\title{
GENERATING FUZZY IMPLICATIONS BY ORDINAL SUMS
}

\author{
PaWę Drygaś - AnNa Król
}

\begin{abstract}
This paper deals with ordinal sums of fuzzy implications. Some of the known constructions are recalled and new ways of generating fuzzy implications from given ones are proposed. Sufficient properties of fuzzy implications as summands for obtaining a fuzzy implication as a result are presented.
\end{abstract}

\section{Introduction}

Fuzzy implications find applications in many fields such as fuzzy control, approximate reasoning, and decision support systems. For that reason, new families of these connectives are the subject of investigation.

One of the directions of such a research is to consider an ordinal sum of fuzzy implications on the pattern of the ordinal sum of t-norms. Some interesting results connected to representation of the residual implication corresponding to a fuzzy conjunction (for example continuous or at least left-continuous tnorm) given by an ordinal sum were obtained in [2], 4], 7]. In [9] $\mathrm{S} \mathrm{u}$ et al. introduced a concept of an ordinal sum of fuzzy implications similar to the construction of the ordinal sum of t-norms. In [3, there were proposed other constructions of ordinal sums of fuzzy implications. In this contribution, some of the ideas are recalled and new possibilities of defining ordinal sums of fuzzy implications are proposed.

First, in Section 2, we recall definitions and basic results concerning t-norms and fuzzy implications and we revise methods of constructing ordinal sums of fuzzy implications. Then, in Section 3, we propose new constructions of ordinal sums of fuzzy implication which generate fuzzy implications. Next, we suggest further research directions for the ordinal sums of fuzzy implications.

(C) 2016 Mathematical Institute, Slovak Academy of Sciences. 2010 Mathematics Subject Classification: 03E72, 06 F05.

Keyw ords: fuzzy implication, ordinal sum, triangular norm.

Partially supported by the Centre for Innovation and Transfer of Natural Sciences and Engineering Knowledge in Rzeszów, through the Project Number RPPK.01.03.00-18-001/10. 


\section{PAWEL DRYGAŚ - ANNA KRÓL}

\section{Basic notions}

Here we recall the notions of a t-norm and a fuzzy implication, as well as some of the constructions of ordinal sums of these fuzzy connectives.

\subsection{Fuzzy connectives}

First, we recollect the definition of a t-norm and an important class of t-norms.

Definition 2.1 ([6, p. 4]). A t-norm is an increasing, commutative and associative operation $T:[0,1]^{2} \rightarrow[0,1]$ with neutral element 1 .

Definition 2.2 ([6, p. 27]). A t-norm $T$ is called Archimedean if for each $(x, y) \in(0,1)^{2}$ there exists $n \in \mathbb{N}$ such that $x_{T}^{(n)}<y$.

ExAmple 2.3 ([6, p. 4], [5, p. 7]). Here, we list well-known t-norms, from which $T_{M}$ is continuous, and $T_{P}, T_{L}$ are both continuous and Archimedean.

$$
\begin{aligned}
& T_{M}(x, y)=\min (x, y), \quad T_{P}(x, y)=x y, \\
& T_{L}(x, y)=\max (x+y-1,0), \quad T_{D}(x, y)= \begin{cases}x & \text { if } y=1, \\
y & \text { if } x=1, \\
0, & \text { otherwise. }\end{cases} \\
& T_{n M}(x, y)= \begin{cases}0 & \text { if } x+y \leq 1, \\
\min (x, y), & \text { otherwise }\end{cases}
\end{aligned}
$$

Next, let us recall a representation of continuous t-norms by means of ordinal sums which is based on the ordinal sum of arbitrary t-norms [6, p. 82].

Theorem 2.4 ([6, p. 128]). For an operation $T:[0,1]^{2} \rightarrow[0,1]$ the following statements are equivalent:

(i) $T$ is a continuous t-norm.

(ii) $T$ is uniquely representable as an ordinal sum of continuous Archimedean t-norms, i.e., there exists a uniquely determined (finite or countably infinite) index set I, a family of uniquely determined pairwise disjoint open subintervals $\left(a_{k}, b_{k}\right)$ of $[0,1]$ and a family of uniquely determined continuous Archimedean t-norms $\left(T_{k}\right)_{k \in A}$ such that (see Fig. 1)

$$
T(x, y)= \begin{cases}a_{k}+\left(b_{k}-a_{k}\right) T_{k}\left(\frac{x-a_{k}}{b_{k}-a_{k}}, \frac{y-a_{k}}{b_{k}-a_{k}}\right) & \text { if }(x, y) \in\left[a_{k}, b_{k}\right]^{2}, \\ \min (x, y), & \text { otherwise }\end{cases}
$$

Now, we focus on fuzzy implications, including the class of R-implications. 


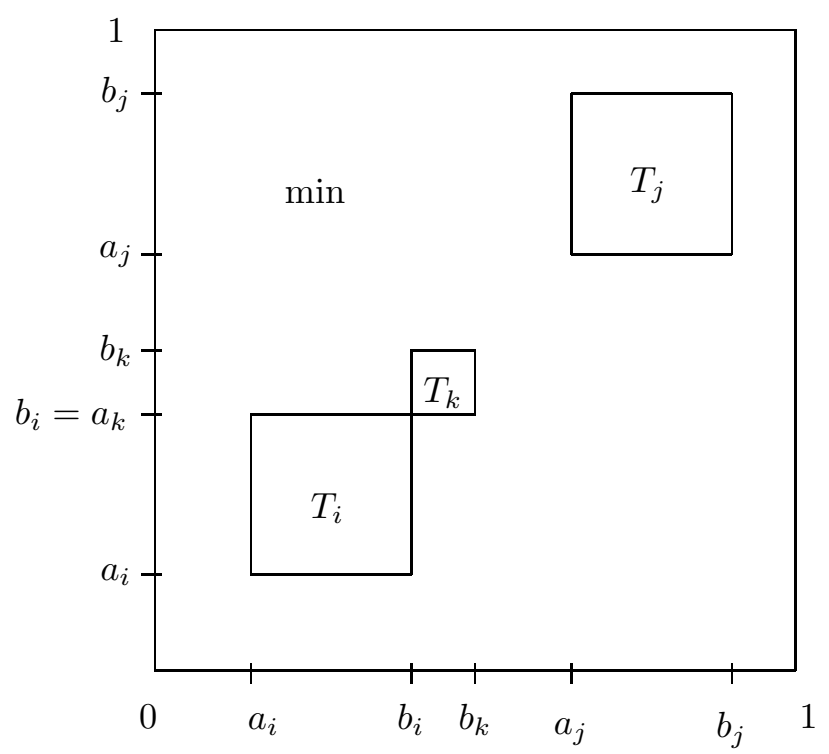

FIgURE 1. The structure of an ordinal sum of t-norms.

Definition 2.5 ([1, p. 2], [5, p. 21]). A function $I:[0,1]^{2} \rightarrow[0,1]$ is called a fuzzy implication if it satisfies the following conditions:

(I1) decreasing in its first variable,

(I2) increasing in its second variable,

(I3) $I(0,0)=1$,

(I4) $I(1,1)=1$,

(I5) $I(1,0)=0$.

There are many potential properties of fuzzy implications (see, e.g., [1, p. 9]). We recall here only one which will be important in the sequel.

Definition 2.6 ([] $]$ ). We say that a fuzzy implication $I$ fulfils the consequent boundary property (CB) if

$$
I(x, y) \geq y, \quad x, y \in[0,1] .
$$

ExAmple 2.7 ([1, pp. 4,5]). The following are well-known fuzzy implications. Almost all of them except for $I_{\mathrm{RS}}$ fulfil property (CB). 


$$
\begin{array}{ll}
I_{\mathrm{EK}}(x, y)=\min (1-x+y, 1), & I_{\mathrm{GG}}(x, y)= \begin{cases}1 & \text { if } x \leq y, \\
\frac{y}{x} & \text { if } x>y,\end{cases} \\
I_{\mathrm{GD}}(x, y)= \begin{cases}1 & \text { if } x \leq y, \\
y & \text { if } x>y,\end{cases} & I_{\mathrm{RS}}(x, y)= \begin{cases}1 & \text { if } x \leq y, \\
0 & \text { if } x>y,\end{cases} \\
I_{\mathrm{RC}}(x, y)=1-x+x y, & I_{\mathrm{YG}}(x, y)= \begin{cases}1 & \text { if } x=0 \text { and } y=0, \\
y^{x} & \text { if else, }\end{cases} \\
I_{\mathrm{DN}}(x, y)=\max (1-x, y), & I_{\mathrm{FD}}(x, y)= \begin{cases}1 & \text { if } x \leq y, \\
\max (1-x, y) & \text { if } x>y,\end{cases} \\
I_{\mathrm{WB}}(x, y)=\left\{\begin{array}{lll}
1 & \text { if } x \leq 1, \\
y & \text { if } x=1, & \text { if } x=1,
\end{array}\right. & I_{\mathrm{DP}}(x, y)= \begin{cases}y-x & \text { if } y=0, \\
1 & \text { if } x<1, y>0 .\end{cases}
\end{array}
$$

Definition 2.8. A function $I:[0,1]^{2} \rightarrow[0,1]$ is called a residual implication (an R-implication) if there exists a t-norm $T$ such that

$$
I(x, y)=I_{T}(x, y)=\sup \{t \in[0,1]: T(x, t) \leq y\}, \quad x, y \in[0,1] .
$$

ExAmple 2.9. Table 1 shows R-implications obtained by the formula (1) from basic t-norms presented in Example 2.3 .

TABLE 1. Examples of basic R-implications.

\begin{tabular}{|c|c|}
\hline t-norm $T$ & R-implication $I_{T}$ \\
\hline$T_{M}$ & $I_{G D}$ \\
\hline$T_{P}$ & $I_{G G}$ \\
\hline$T_{L}$ & $I_{L K}$ \\
\hline$T_{D}$ & $I_{W B}$ \\
\hline$T_{n} M$ & $I_{F D}$ \\
\hline
\end{tabular}

Theorem 2.10 ([1, p. 83]). If $T$ is a continuous t-norm with an ordinal sum structure (see Theorem 2.4), then the corresponding $R$-implication $I_{T}$ is given by (see Fig. Q) 


$$
I_{T}(x, y)= \begin{cases}1 & \text { if } x \leq y \\ a_{k}+\left(b_{k}-a_{k}\right) I_{T_{k}}\left(\frac{x-a_{k}}{b_{k}-a_{k}}, \frac{y-a_{k}}{b_{k}-a_{k}}\right) & \text { if } x, y \in\left[a_{k}, b_{k}\right], x>y \\ y, & \text { otherwise }\end{cases}
$$
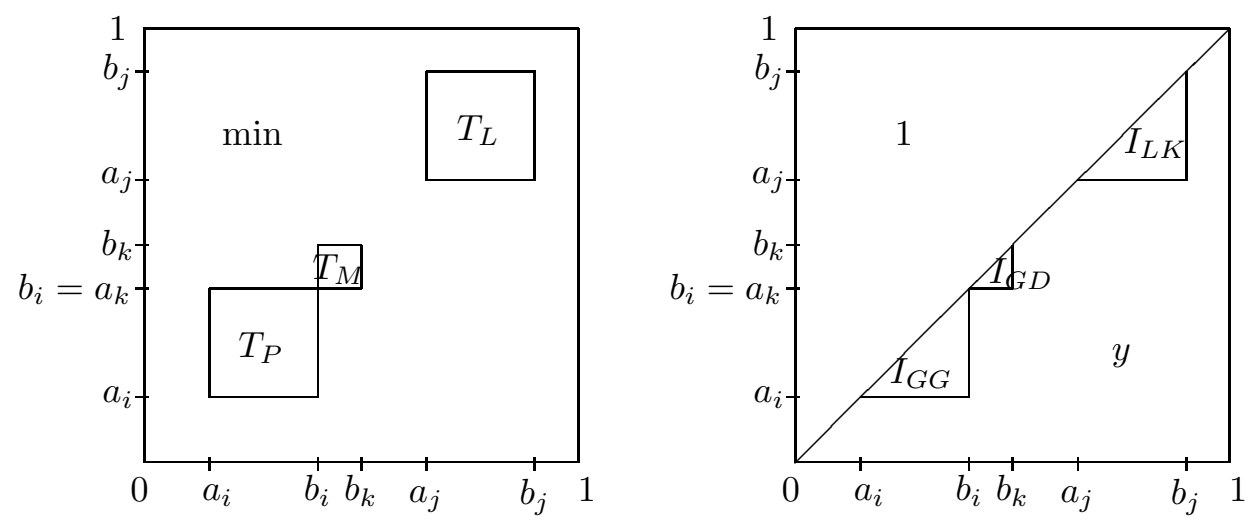

Figure 2. The structures of an ordinal sum of t-norms and R-implication $I_{T}$ given by (2).

\subsection{Ordinal sums of fuzzy implications}

Now, let us recall a recent approach to the construction of ordinal sum of fuzzy implications. Let start with the method based on the construction of the ordinal sum of t-norms.

Definition 2.11 (9]). Let $\left\{I_{k}\right\}_{k \in A}$ be a family of implications and $\left\{\left[a_{k}, b_{k}\right]\right\}_{k \in A}$ be a family of pairwise disjoint close subintervals of [0,1] with $0<a_{k}<b_{k}$ for all $k \in A$, where $A$ is a finite or countably infinite index set. The operation $I:[0,1]^{2} \rightarrow[0,1]$ given by (see Fig. 3)

$$
I(x, y)= \begin{cases}a_{k}+\left(b_{k}-a_{k}\right) I_{k}\left(\frac{x-a_{k}}{b_{k}-a_{k}}, \frac{y-a_{k}}{b_{k}-a_{k}}\right) & \text { if } x, y \in\left[a_{k}, b_{k}\right], \\ I_{G D}(x, y), & \text { otherwise, }\end{cases}
$$

is called an ordinal sum of fuzzy implications $\left\{I_{k}\right\}_{k \in A}$.

The following theorem characterizes the ordinal sum $I$ given by (3) as fuzzy implication.

Theorem $2.12(9])$. Let $\left\{I_{k}\right\}_{k \in A}$ be a family of fuzzy implications. The operation $I$ given by (3) is a fuzzy implication if and only if $I_{k}$ satisfies (CB) whenever $k \in A$ and $b_{k}<1$. 
PAWEL DRYGAŚ - ANNA KRÓL

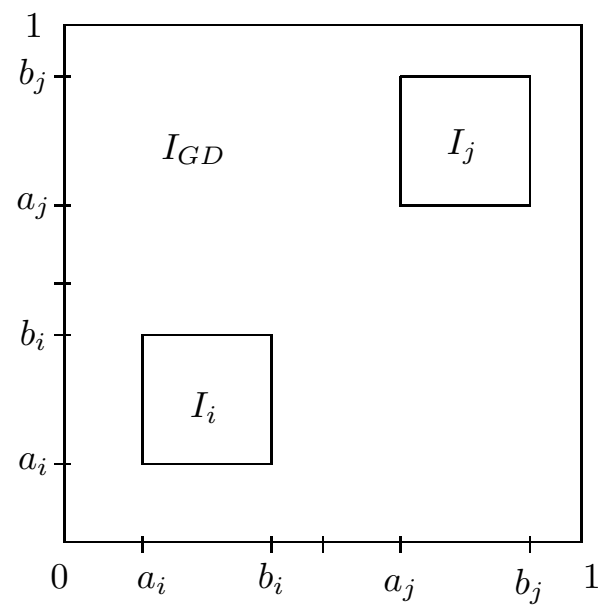

Figure 3. The structure of an ordinal sum of fuzzy implications given by (3).

The following method is a kind of generalization of the results obtained, e.g., in [7] for residual implications.

Definition 2.13 ([3]). Let $\left\{I_{k}\right\}_{k \in A}$ be a family of implications and $\left\{\left(a_{k}, b_{k}\right)\right\}_{k \in A}$ be a family of pairwise disjoint subintervals of $[0,1]$ with $a_{k}<b_{k}$ for all $k \in A$, where $A$ is a finite or countably infinite index set. Let us consider an operation $I:[0,1]^{2} \rightarrow[0,1]$ given by the following formula (see Fig. 4)

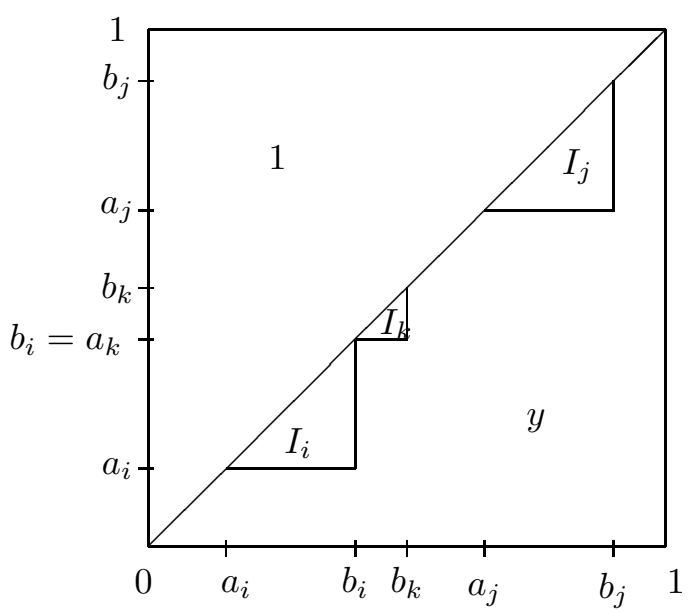

Figure 4. The structure of an operation given by (4). 


$$
I(x, y)= \begin{cases}a_{k}+\left(b_{k}-a_{k}\right) I_{k}\left(\frac{x-a_{k}}{b_{k}-a_{k}}, \frac{y-a_{k}}{b_{k}-a_{k}}\right) & \text { if } x, y \in\left[a_{k}, b_{k}\right], x>y \\ I_{G D}(x, y), & \text { otherwise }\end{cases}
$$

Similarly to the previous methods, we have the result characterizing the ordinal sum $I$ given by (4) as fuzzy implication.

Theorem 2.14 ([3]). Let $\left\{I_{k}\right\}_{k \in A}$ be a family of fuzzy implications. The operation $I$ given by (4) is a fuzzy implication if and only if $I_{k}$ satisfies (CB) whenever $k \in A$ and $b_{k}<1$.

As we can see, not every fuzzy implication can be used in constructions (3) and (4). Below we recall a structure in which any fuzzy implications can be used.

Definition 2.15 ([3]). Let $\left\{I_{k}\right\}_{k \in A}$ be a family of implications and $\left\{\left[a_{k}, b_{k}\right]\right\}_{k \in A}$ be a family of pairwise disjoint close subintervals of $[0,1]$ with $0<a_{k}<b_{k}$ for all $k \in A$, where $A$ is a finite or countably infinite index set. Let us consider an operation $I:[0,1]^{2} \rightarrow[0,1]$ given by the following formula (see Fig. 5)

$$
I(x, y)= \begin{cases}a_{k}+\left(b_{k}-a_{k}\right) I_{k}\left(\frac{x-a_{k}}{b_{k}-a_{k}}, \frac{y-a_{k}}{b_{k}-a_{k}}\right) & \text { if } x, y \in\left[a_{k}, b_{k}\right] \\ I_{R S}(x, y), & \text { otherwise }\end{cases}
$$

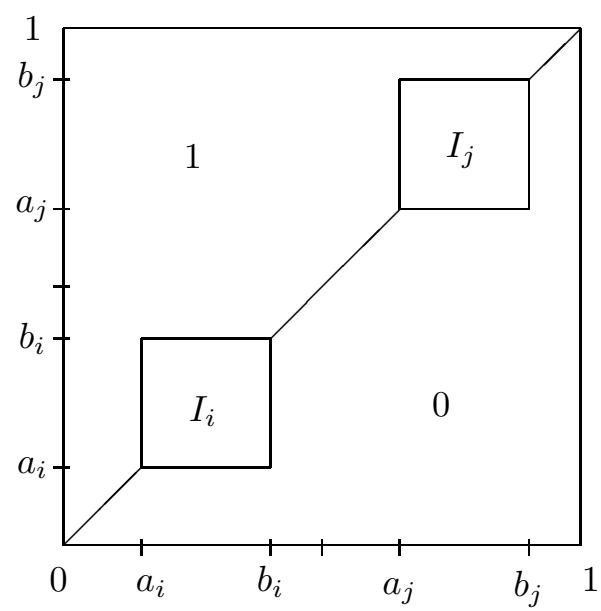

FiguRE 5. The structure of an operation given by (5).

TheOREm 2.16 ([3]). The operation I given by (5]) is a fuzzy implication. 


\section{PAWEL DRYGAŚ - ANNA KRÓL}

\section{Main results}

Let us notice, that all the constructions presented in Section 2.2 involve intervals $\left[a_{i}, b_{i}\right]$ which are necessarily disjoint. This means that we are unable to construct fuzzy implications in which all the values $I(x, x)$ for $x \in(0,1)$ depend on the component implications $I_{k}$. Below we propose two constructions that solve this problem.

Definition 3.1. Let $\left\{I_{k}\right\}_{k \in A}$ be a family of implications and $\left\{\left(a_{k}, b_{k}\right)\right\}_{k \in A}$ be a family of pairwise disjoint subintervals of [0,1] with $a_{k}<b_{k}$ for all $k \in A$, where $A$ is a finite or countably infinite index set. Let us consider an operation $I:[0,1]^{2} \rightarrow[0,1]$ given by the following formula (see Fig. 6)

$$
I(x, y)= \begin{cases}a_{k}+\left(b_{k}-a_{k}\right) I_{k}\left(\frac{x-a_{k}}{b_{k}-a_{k}}, \frac{y-a_{k}}{b_{k}-a_{k}}\right) & \text { if } x, y \in\left(a_{k}, b_{k}\right] \\ I_{R S}(x, y), & \text { otherwise }\end{cases}
$$

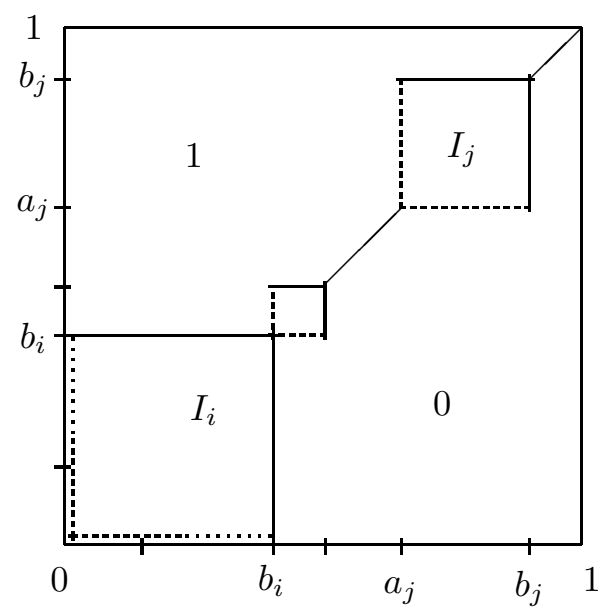

Figure 6. The structure of an operation given by (6).

For the above defined operation we have the following result.

TheOREm 3.2. The operation I given by (6) is a fuzzy implication.

P r o o f. First, let us consider the condition (I1). Let $x_{1}, x_{2}, y \in[0,1], x_{1}<x_{2}$.

If $y \in\left(a_{k}, b_{k}\right]$ for some $k \in A$, then we consider the following cases

1. $x_{1} \leq a_{k}$. Then, $I\left(x_{1}, y\right)=I_{R S}\left(x, y_{1}\right)=1 \geq I\left(x_{2}, y\right)$. 
2. $x_{1}, x_{2} \in\left(a_{k}, b_{k}\right]$. Then, using monotonicity of $I_{k}$ with respect to the first variable we have

$$
I_{k}\left(\frac{x_{1}-a_{k}}{b_{k}-a_{k}}, \frac{y-a_{k}}{b_{k}-a_{k}}\right) \geq I_{k}\left(\frac{x_{2}-a_{k}}{b_{k}-a_{k}}, \frac{y-a_{k}}{b_{k}-a_{k}}\right) .
$$

This means that $I\left(x_{1}, y\right) \geq I\left(x_{2}, y\right)$.

3. $b_{k}<x_{2}$. Then $I\left(x_{1}, y\right) \geq 0=I_{R S}\left(x_{2}, y\right)=I\left(x_{2}, y\right)$.

If $y \notin\left(a_{k}, b_{k}\right]$ for any $k \in A$, then

$$
I\left(x_{1}, y\right)=I_{R S}\left(x_{1}, y\right) \geq I_{R S}\left(x_{2}, y\right)=I\left(x_{2}, y\right),
$$

which gives condition (I1).

To prove (I2) let us take $x, y_{1}, y_{2} \in[0,1], y_{1}<y_{2}$.

If $x \in\left(a_{k}, b_{k}\right]$ for some $k \in A$, then we obtain the following cases

1. $y_{1} \leq a_{k}$. Then $I\left(x, y_{1}\right)=I_{R S}\left(x, y_{1}\right)=0 \leq I\left(x, y_{2}\right)$.

2. $y_{1}, y_{2} \in\left(a_{k}, b_{k}\right]$. Then using monotonicity of $I_{k}$ with respect to the second variable we have

$$
I_{k}\left(\frac{x-a_{k}}{b_{k}-a_{k}}, \frac{y_{1}-a_{k}}{b_{k}-a_{k}}\right) \leq I_{k}\left(\frac{x-a_{k}}{b_{k}-a_{k}}, \frac{y_{2}-a_{k}}{b_{k}-a_{k}}\right) .
$$

This means that $I\left(x, y_{1}\right) \leq I\left(x, y_{2}\right)$.

3. $b_{k}<y_{2}$. Then $I\left(x, y_{1}\right) \leq 1=I_{R S}\left(x, y_{2}\right)=I\left(x, y_{2}\right)$.

If $x \notin\left(a_{k}, b_{k}\right]$ for any $k \in A$, then $I\left(x, y_{1}\right)=I_{R S}\left(x, y_{1}\right) \leq I_{R S}\left(x, y_{2}\right)=I\left(x, y_{2}\right)$. So, we obtain (I2).

Directly from (6) we have $I(0,0)=I_{R S}(0,0)=0$ and $I(1,0)=I_{R S}(1,0)=0$. So $I$ fulfils (I3) and (I5). To prove (I4) let us consider two cases. If there exists $k \in A$ such that $b_{k}=1$, then

$$
I(1,1)=a_{k}+\left(1-a_{k}\right) I_{k}\left(\frac{1-a_{k}}{1-a_{k}}, \frac{1-a_{k}}{1-a_{k}}\right)=a_{k}+\left(1-a_{k}\right) I_{k}(1,1)=1 .
$$

Otherwise $I(1,1)=I_{R S}(1,1)=1$.

So, operation $I$ given by (6) is a fuzzy implication.

EXAMPLE 3.3. Let

$$
I(x, y)= \begin{cases}0.5 I_{D N}(2 x, 2 y) & \text { if } x, y \in(0,0.5] \\ 0.5+0.1 I_{L K}(10 x-5,10 y-5) & \text { if } x, y \in(0.5,0.6] \\ I_{Y G}(x, y), & \text { otherwise }\end{cases}
$$

$I$ is a fuzzy implication generated by the ordinal sum (므).

The other construction is following. 
Definition 3.4. Let $\left\{I_{k}\right\}_{k \in A}$ be a family of implications and $\left\{\left(a_{k}, b_{k}\right)\right\}_{k \in A}$ be a family of pairwise disjoint subintervals of $[0,1]$ with $0<a_{k}<b_{k}$ for all $k \in A$, where $A$ is a finite or countably infinite index set. Let us consider an operation $I:[0,1]^{2} \rightarrow[0,1]$ given by the following formula (see Fig. (7))

$$
I(x, y)= \begin{cases}a_{k}+\left(b_{k}-a_{k}\right) I_{k}\left(\frac{x-a_{k}}{b_{k}-a_{k}}, \frac{y-a_{k}}{b_{k}-a_{k}}\right) & \text { if } x, y \in\left[a_{k}, b_{k}\right), \\ I_{R S}(x, y), & \text { otherwise }\end{cases}
$$

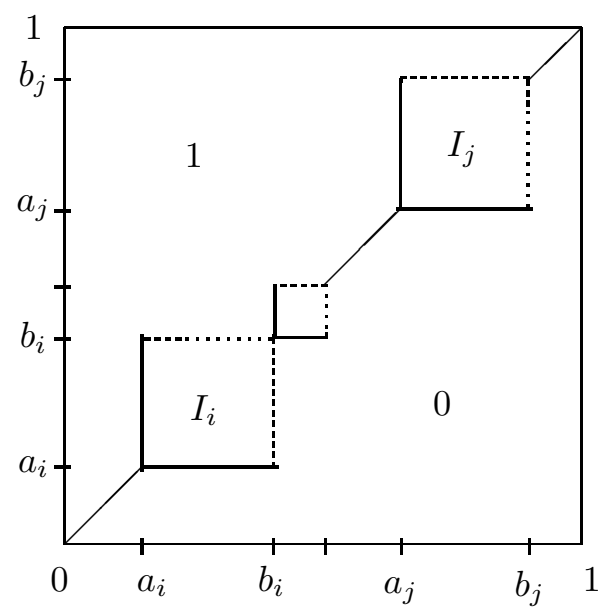

Figure 7. The structure of an operation given by (7).

Similarly as in the previous construction we obtained the following result.

Theorem 3.5. The operation I given by (7) is a fuzzy implication.

Pro of. First, let us consider the condition (I1). Let $x_{1}, x_{2}, y \in[0,1], x_{1}<x_{2}$.

If $y \in\left[a_{k}, b_{k}\right)$ for some $k \in A$, then we consider the following cases

1. $x_{1}<a_{k}$. Then, $I\left(x_{1}, y\right)=I_{R S}\left(x, y_{1}\right)=1 \geq I\left(x_{2}, y\right)$.

2. $x_{1}, x_{2} \in\left[a_{k}, b_{k}\right)$. Then, using monotonicity of $I_{k}$ with respect to the first variable we have

$$
I_{k}\left(\frac{x_{1}-a_{k}}{b_{k}-a_{k}}, \frac{y-a_{k}}{b_{k}-a_{k}}\right) \geq I_{k}\left(\frac{x_{2}-a_{k}}{b_{k}-a_{k}}, \frac{y-a_{k}}{b_{k}-a_{k}}\right) .
$$

This means that $I\left(x_{1}, y\right) \geq I\left(x_{2}, y\right)$.

3. $b_{k} \leq x_{2}$. Then

$$
I\left(x_{1}, y\right) \geq 0=I_{R S}\left(x_{2}, y\right)=I\left(x_{2}, y\right) .
$$

If $y \notin\left[a_{k}, b_{k}\right)$ for any $k \in A$, then

$$
I\left(x_{1}, y\right)=I_{R S}\left(x_{1}, y\right) \geq I_{R S}\left(x_{2}, y\right)=I\left(x_{2}, y\right),
$$

which gives condition (I1). 


\section{GENERATING FUZZY IMPLICATIONS BY ORDINAL SUMS}

To prove (I2) let us take $x, y_{1}, y_{2} \in[0,1], y_{1}<y_{2}$.

If $x \in\left[a_{k}, b_{k}\right)$ for some $k \in A$, then we obtain the following cases

1. $y_{1}<a_{k}$. Then $I\left(x, y_{1}\right)=I_{R S}\left(x, y_{1}\right)=0 \leq I\left(x, y_{2}\right)$.

2. $y_{1}, y_{2} \in\left[a_{k}, b_{k}\right)$. Then using monotonicity of $I_{k}$ with respect to the second variable we have

$$
I_{k}\left(\frac{x-a_{k}}{b_{k}-a_{k}}, \frac{y_{1}-a_{k}}{b_{k}-a_{k}}\right) \leq I_{k}\left(\frac{x-a_{k}}{b_{k}-a_{k}}, \frac{y_{2}-a_{k}}{b_{k}-a_{k}}\right) .
$$

This means that $I\left(x, y_{1}\right) \leq I\left(x, y_{2}\right)$.

3. $b_{k} \leq y_{2}$. Then $I\left(x, y_{1}\right) \leq 1=I_{R S}\left(x, y_{2}\right)=I\left(x, y_{2}\right)$.

If $x \notin\left[a_{k}, b_{k}\right)$ for any $k \in A$, then $I\left(x, y_{1}\right)=I_{R S}\left(x, y_{1}\right) \leq I_{R S}\left(x, y_{2}\right)=I\left(x, y_{2}\right)$. So, we obtain (I2).

Directly from (7) we have $I(0,0)=I_{R S}(0,0)=1, I(1,1)=I_{R S}(1,1)=1$ and $I(1,0)=I_{R S}(1,0)=0$. Therefore $I$ fulfils (I3), (I4) and (I5). So, operation $I$ given by (7) is a fuzzy implication.

EXAMPLE 3.6. Let

$$
I(x, y)= \begin{cases}0.5 I_{R C}(5 x-1,5 y-1) & \text { if } x, y \in[0.2,0.4) \\ 0.5+0.1 I_{L K}(10 x-5,10 y-5) & \text { if } x, y \in(0.5,0.6] \\ I_{R S}(x, y), & \text { otherwise }\end{cases}
$$

$I$ is a fuzzy implication generated by the ordinal sum (7).

\section{Conclusion}

In this paper, we presented methods of constructing ordinal sums of fuzzy implications. Moreover, we examined whether the ordinal sums are fuzzy implications. In future research, it would be useful to discuss properties of these ordinal sums. Another problem is whether the proposed ordinal sums preserve properties of its summands.

\section{REFERENCES}

[1] BACZYŃSKI, M.-JAYARAM, B.: Fuzzy Implications, in: Stud. Fuzziness Soft Comput., Vol. 231, Springer, Berlin, 2008.

[2] DE BAETS, B.-MESIAR, R.: Residual implicators of continuous t-norms, in: Proc. of 4th European Congress on Intelligent Techniques and Soft Computing-EUFIT '96 (H. J. Zimmermann, ed.), Vol. 1, Verlag Mainz, Aachen, Germany, 1996, pp. 27-31. 


\section{PAWEL DRYGAŚ - ANNA KRÓL}

[3] DRYGAŚ, P.-KRÓL, A.: Various kinds of ordinal sums of fuzzy implications, in: Novel Developments in Uncertainty Represent. and Processing, (K. T. Atanassov et al., eds.), Advances in Intelligent Systems and Computing, Vol. 401, Springer Internat. Publ. AG Switzerland, 2016, pp. 37-49.

[4] DURANTE, F.-KLEMENT, E. P.-MESIAR, R.-SEMPI, C.: Conjunctors and their residual implicators: characterizations and construction methods, Mediterr. J. Math. 4 (2007), 343-356.

[5] FODOR, J.-ROUBENS, M.: Fuzzy Preference Modelling and Multicriteria Decision Support, Kluwer Acad. Publ., Dordrecht, 1994.

[6] KLEMENT, E. P.-MESIAR, R.-PAP, E.: Triangular Norms, Kluwer Acad. Publ., Dordrecht, 2000.

[7] MESIAR, R.-MESIAROVA, A.: Residual implications and left-continuous t-norms which are ordinal sums of semigroups, Fuzzy Sets and Systems 143 (2004), 47-57.

[8] SHI, Y.-VAN GASSE, B.-RUAN, D.-KERRE, E.: On dependencies and independencies of fuzzy implication axioms, Fuzzy Sets and Systems 161 (2010), 1388-1405.

[9] SU, Y.-XIE, A.-LIU, H.: On ordinal sum implications, Inform. Sci. 293 (2015), $251-262$.

Received July 15, 2016

Interdisciplinary Centre for Computational Modelling

Faculty of Mathematics and Natural Sciences

University of Rzeszów

Pigonia 1

PL-35-310 Rzeszów

POLAND

E-mail: paweldrs@ur.edu.pl annakrol@ur.edu.pl 